\title{
Using surface-plasmon effects to improve process latitute in near-field optical lithography
}

\author{
Matthew D Arnold and Richard J Blaikie* \\ MacDiarmid Institute for Advanced Materials and Nanotechnology \\ Department of Electrical and Computer Engineering \\ University of Canterbury, Christchurch 8020, New Zealand \\ *Email: r.blaikie@elec.canterbury.ac.nz \\ Telephone: +64 (3) 364 7001, Fax: +64 (3) 3642761
}

\begin{abstract}
Surface Plasmon Enhanced Contact Lithography uses an underlying plasmonic layer to enhance image quality in Evanescent Near-Field Optical Lithography. This article details simulations aimed at finding optimum conditions and attempts to explain some underlying mechanisms. Parameters explored include resist thickness, metal permittivity and thickness, polarization, and grating period and duty-cycle.
\end{abstract}

Keywords- nanolithography; surface plasmons; photoresist; electromagnetic simulations

\section{INTRODUCTION}

The resolution limits of projection optical lithography can be overcome by working in the evanescent near-field regionsomething we have termed Evanescent Near-Field Optical Lithography (ENFOL) - and there have been a number of demonstrations of nanometer-scale-resolution pattering using this method [1-3]. In essence this is an adaptation of well established contact lithography techniques, so it offers the ability to pattern large-area substrates with nanometer-scale features quickly and easily using conventional processes.

Surface Plasmon Enhanced Contact Lithography (SPECL) is a modified form of ENFOL designed to improve subwavelength image quality by placing a noble metal beneath the imaging layer in the near-field of a mask. Previous computational $[4,5]$ and experimental [6] studies suggest that that SPECL can improve process latitude compared to pure contact techniques, but future investigations will benefit from a better understanding of the underlying mechanism.

\section{PRINCIPLES OF SPECL}

Fig. 1 outlines the principles of ENFOL and SPECL, showing finite element electromagnetic simulations of where the image is formed and how a noble metal layer is employed to improve the process latitude conditions. In the ENFOL case of Fig. 1(a) the depth of field (DOF) of the image is limited and the wide separation of the $\pm 10 \%$ iso-intensity contours show that the imaging is extremely sensitive to small changes in exposure or development conditions.

In contrast, the SPECL image of Fig. 1(b) extends uniformly throughout the whole resist layer, and the closelyspaced iso-intensity contours indicate that the process latitude should be very good. We interpret the results in terms of balanced interference of the "sources" consisting of the mask at the top and coupled plasmons induced on the metal layer at the bottom. Reflected evanescent fields are amplified by a factor that increases with the lateral propagation and hence the usual "phase" decay is partially compensated.
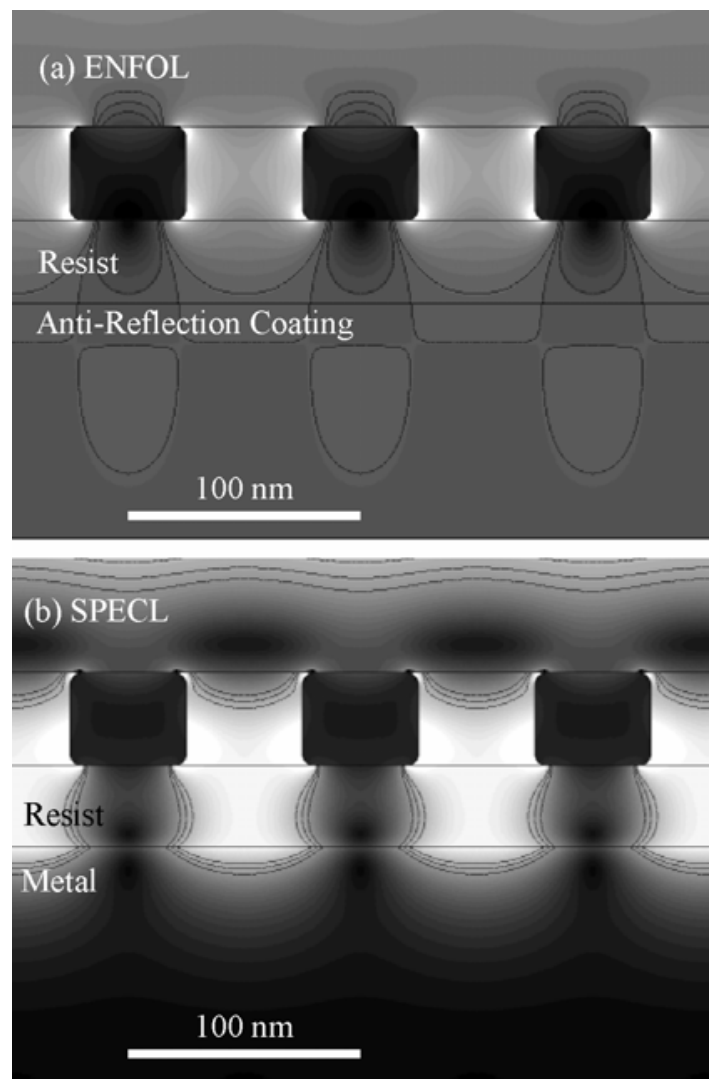

Figure 1. Two-dimensional finite element simulations of (a) ENFOL and (b) SPECL exposures of a $100-\mathrm{nm}$ period grating with $365 \mathrm{~nm}$ wavelength illumination. The principal difference between the two methods is that ENFOL uses an index-matched (anti-reflection) layer beneath the resist, whereas SPECL employs a highly mismatched metallic intermediate layer. The intensities are shown on a linear scale from white (high intensity) to black (zero), and $\pm 10 \%$ iso-intensity contours are shown together with the intensity contour for the best image in the resist. 


\section{SCOPE AND METHODS}

This study investigates the improvements in process performance that should be achievable in a SPECL scheme, targeting sub-100 $\mathrm{nm}$ resolution into $50-\mathrm{nm}$ thick photoresist layers, with the aim to determine the optimal system geometry and resultant process conditions for UV exposure $(365 \mathrm{~nm}$ illumination) with silver as the underlying metal layer. Silver was chosen because of its ability to support strong surface plasmon modes around $365 \mathrm{~nm}$, and due to the fact that it is the material of choice for related plasmonic superlensing phenomena that have recently been reported [7].

The comprehensive simulations described in this paper use finite element analysis on simple periodic grating structures, with perfectly-matched-layers as free-space terminators and surface electric and magnetic current sources to simulate farfield plane-waves at normal incidence. Virtually all simulated parameters were based on experimentally accessible values, with fixed parameters as detailed in Table I. Other values are as detailed in Table II, unless otherwise stated.

\begin{tabular}{|l|l|}
\multicolumn{1}{c}{ TABLE I. FIXED PARAMETERS } \\
\begin{tabular}{|l|l|}
\hline \multicolumn{1}{|c|}{ Parameter } & \multicolumn{1}{c|}{ Value } \\
\hline Wavelength & $365 \mathrm{~nm}$ \\
\hline Cover permittivity & 2.25 (Glass) \\
\hline Mask thickness & $40 \mathrm{~nm}$ \\
\hline Mask corner radii & $5 \mathrm{~nm}$ \\
\hline Mask aperture permittivity & 1.0 (Air) \\
\hline Mask absorber permittivity & $4.4-18.0 \mathrm{i}$ (Tungsten) \\
\hline Resist Permittivity & 2.9 \\
\hline Substrate Permittivity & 2.9 \\
\hline
\end{tabular}
\end{tabular}

TABLE II. DEFAULT PARAMETERS

\begin{tabular}{|l|l|}
\hline \multicolumn{1}{|c|}{ Parameter } & \multicolumn{1}{c|}{ Value } \\
\hline Polarization & TM \\
\hline Grating period & $100 \mathrm{~nm}$ \\
\hline Grating absorber width & $50 \mathrm{~nm}$ \\
\hline Metal thickness & $100 \mathrm{~nm}$ \\
\hline Metal permittivity & -2.9 \\
\hline
\end{tabular}

\section{Contrast And Depth-OF-Field}

The relationship between contrast and depth-of-field (DOF) is very important in near-field lithography, which was clearly demonstrated in Fig. 1(a) where the visibility of the ENFOL image decays rapidly with distance from the mask. Representative examples showing the relationship between DOF and contrast are plotted for ENFOL and SPECL in Fig. 2. In this study we have defined the contrast (for a given depth of field) as:

$$
\text { Contrast }=\left(I_{1} / I_{0}\right)-1
$$

where the intensities are defined in terms of target areas such that $I_{1}$ is the minimum with depth for the laterally averaged intensity under the mask aperture, and $I_{0}$ is the maximum with depth for the laterally averaged intensity under the mask absorber. The improvement in contrast at a fixed DOF or vice versa is evident in Fig. 2, and we now define a merit product

$$
\text { Merit Product }=\text { Contrast } \times \mathrm{DOF}^{2}
$$

that emphasizes the importance of DOF in lithography exposures. The merit product is a quantitative measure of the image quality that we can now use to optimize the SPECL system parameters.

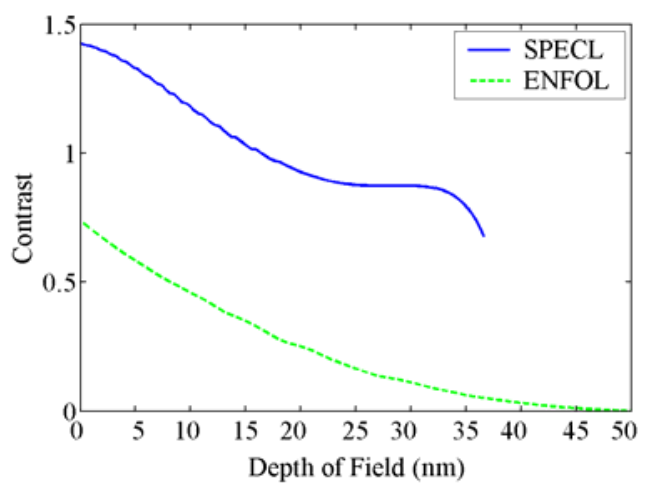

Figure 2. Contrast versus depth of field for ENFOL and SPECL images for a grating of $100 \mathrm{~nm}$ period, $50 \%$ duty cycle.

\section{COUPLING DisTANCE AND INTERFERENCE}

A fundamental feature of SPECL is interference between forward and reflected waves potentially leading to oscillation with resist thickness; thus it is important to optimize this parameter. The optimal separation between the mask and the underlying metal layer can be determined from simulations such as those shown in Fig. 3.

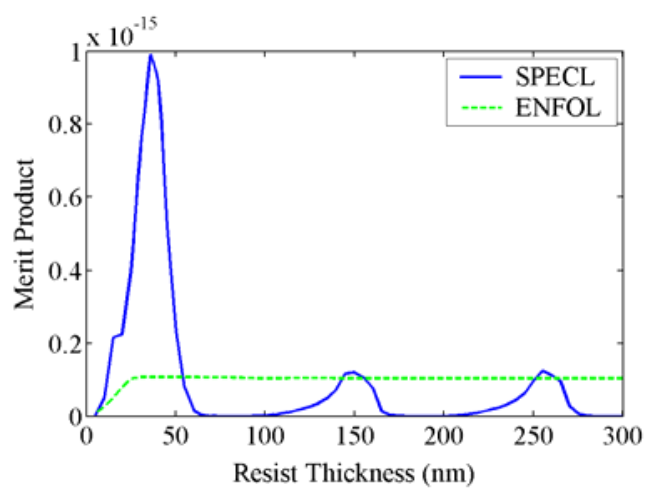

Figure 3. Merit product versus resist thickness for ENFOL and SPECL images for gratings of $100 \mathrm{~nm}$ period, $50 \%$ duty cycle. 
In this particular case the Merit Product peaks for a $35 \mathrm{~nm}$ separation; at this separation there is optimal coupling into the surface plasmon modes on the underlying silver, whilst retaining uniform exposure throughout the resist layer. Simulations consistently show that at larger separations the coupling to the plasmon is relatively weak, resulting in reduced contrast near the metal. Classical standing-wave interference is also evident at larger distances, with oscillations occurring at half-wavelength intervals. It should be noted from Fig. 3 that the Merit Product for SPECL falls well below that for ENFOL at non-optimal spacer thicknesses, so care must be taken when using this technique.

\section{PERMITTIVITY OPTIMIZATION}

The optical properties of the metal layer are of considerable importance in transmission geometries (i.e. superlensing) and so we have investigated the corresponding effect on SPECL. The peak merit product was determined as a function of the permittivity of the metal, as shown in the example in Fig. 4.

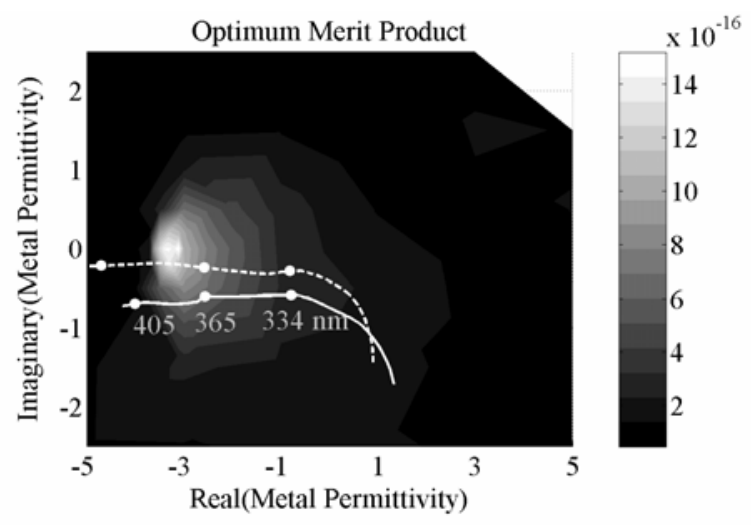

Figure 4. Peak merit product as a function of metal permittivity for a grating of $100 \mathrm{~nm}$ period, $50 \%$ duty cycle, assuming photoresist permittivity 2.9 . For comparison, the merit product achieved by ENFOL is only $1 \times 10-16$. Two different curves for silver permittivity are overlaid in white: Johnson \& Christy [8] (dashed), Palik [9] (solid).

Maximum effect was found when the silver permittivity has the negative real part with magnitude matching that of the photoresist which has positive permittivity. Ideally any loss in the photoresist (negative imaginary permittivity) would be compensated by corresponding gain in the underlying layer, but note that causality should be carefully considered in any such interpretation. Fortunately most photoresist operates in a low-loss regime and Fig. 4 shows that SPECL has some tolerance to mismatch. The simulation in Fig. 4 was repeated for other grating periods and the overall behavior was the same, except that smaller periods resulted in reduced tolerance. Experimental feasibility is also demonstrated in Fig. 4 by comparison to two different silver spectra $[8,9]$, overlaid in white with dots corresponding to 405,365 , and $334 \mathrm{~nm}$ from left-to-right. Clearly silver is ideal as it has low-loss and the $365 \mathrm{~nm}$ "i-line" is well-placed to capture SPECL in conventional photoresist.

\section{POLARIZATION}

Throughout this paper it is tacitly assumed that TM polarization is desirable to allow plasmon coupling, however it is worth examining the actual polarization behavior. Figure 5 shows examples of linearly polarized and unpolarized responses, including both SPECL and ENFOL.

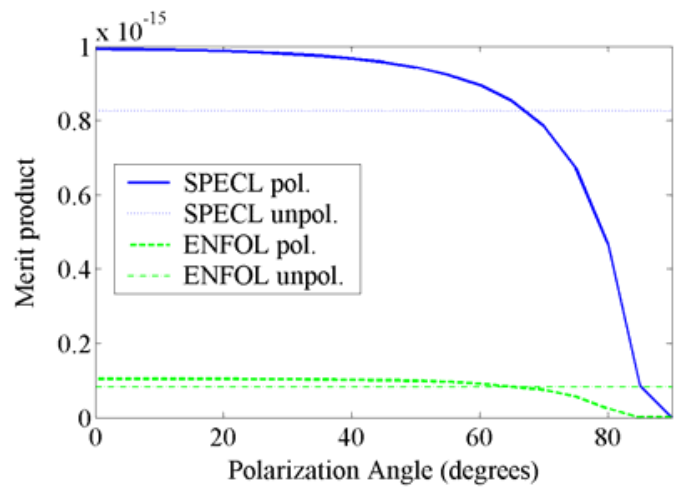

Figure 5. Merit product versus polarization for ENFOL and SPECL images.

It is clear from Fig. 5 that whilst pure TM polarization is optimal, SPECL offers improvements over ENFOL at most polarizations and image quality remains high until polarization is nearly pure TE. The relatively robust response to polarization is explained by the low intensity of the TE component which is usually overwhelmed by the TM component. An important consequence of this finding is that an unpolarized source can be used to simultaneously expose lines of any orientation.

\section{METAL THICKNESS}

Another issue of practical importance is the thickness of metal required, as the metal-substrate interface contributes another reflection that may disturb the SPECL effect. Fig. 6 shows the typical behavior as metal thickness is increased. Initially response is low, and decreases somewhat before rising rapidly, overshooting slightly and then settling towards the value for infinite thickness.

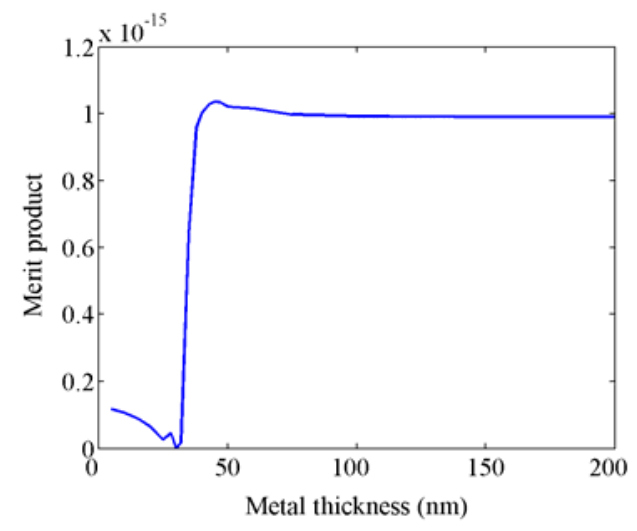

Figure 6. Merit product as a function of metal thickness for a grating of $100 \mathrm{~nm}$ period, $50 \%$ duty cycle, assuming metal permittivity -2.9 . 
We have found that $100 \mathrm{~nm}$ adequately approximates infinite behavior for the parameter scope of this paper; the evanescent components have decayed enough that they do not reach the second interface. The thickness required to achieve usable response varies somewhat depending on system parameters: $40 \mathrm{~nm}$ is usually sufficient and it seems that less may be required for smaller features due to the more rapid decay of the image.

\section{GeOMETRICAL EFFECTS}

A particularly important consideration is the effect of system parameters on the optimum coupling distance (i.e. resist thickness). Extensive simulations reveal that the optimum distance does depend on absorber width but is virtually independent of period, as seen in Fig. 7.

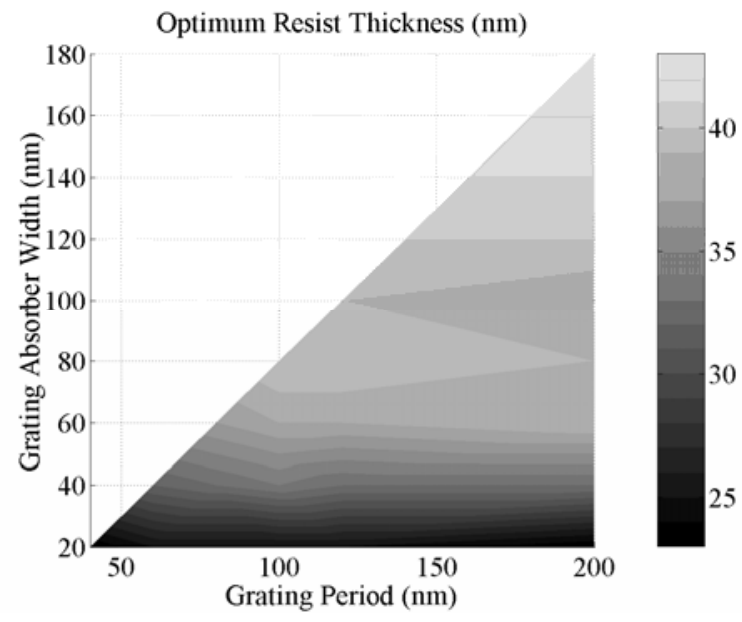

Figure 7. Optimum coupling distance as a function of grating period and absorber width, assuming metal permittivity -2.9 .

The optimal distance is not particularly sensitive to geometric factors, except for narrow absorbers that require closer coupling to achieve the correct balance of highfrequency components. The optimal distance scale is dependent on permittivity but the overall geometric trend is preserved in the general situation. We are currently investigating the reasons behind this apparently universal behavior.

\section{CONCLUSION}

These simulation results show that there is strong merit in using a metallic mismatched intermediate layer in near-field exposures - something that is counter-intuitive to the notion that good index matching is desirable to prevent substrate reflections in photolithography. The improvements that can be achieved are very significant, and the inclusion of a metallic intermediate layer may be compatible with many modern bilayer and tri-layer resist schemes.

\section{ACKNOWLEDGMENT}

This work is supported by the Marsden Fund administered by the Royal Society of New Zealand (contract UOC-312).

\section{REFERENCES}

[1] M. M. Alkaisi, R. J. Blaikie, S. J. McNab, R. Cheung and D. R. S. Cumming, "Sub-diffraction-limited patterning using evanescent nearfield optical lithography," Appl. Phys. Lett. 75, 3560-3562 (1999).

[2] H. Schmid, H. Biebuyck, B. Michel and O.J.F. Martin, "Light-coupling masks for lensless, sub-wavelength optical lithography," Appl. Phys. Lett. 72, 2379-2381 (1998).

[3] J.G. Goodberlet and H. Kavak, "Patterning sub-50 nm features with near-field embedded-amplitude masks," Appl. Phys. Lett. 81, 1315-1317 (2002).

[4] R.J. Blaikie, M.M. Alkaisi, S.J. McNab, S.J. and D.O.S. Melville, "Nanoscale optical patterning using evanescent fields and surface plasmons" International Journal of Nanoscience 3 (nos 4-5), 405-17 (2004)

[5] D.B. Shao, and S.C. Chen, "Surface-plasmon-assisted nanoscale photolithography by polarised light" Appl. Phys. Lett. 86, 253107-1-3 (2005).

[6] D.B. Shao, and S.C. Chen, "Numerical simulation of surface-plasmonassisted nanolithography,” Opt. Express 13, 6964-6973 (2005).

[7] D. O. S. Melville and R. J. Blaikie "Super-resolution imaging through a planar silver layer," Opt. Express 13, 2127-2134 (2005).

[8] P. B. Johnson and R. W. Christy, "Optical Constants of the Noble Metals”, Phys. Rev. B 6, 4370-4379 (1972).

[9] Palik, Handbook of Optical Constants, Academic Press, 1985. 